\title{
Analysis of the asymmetrically expressed Ablim 1 locus reveals existence of a lateral plate Nodal-independent left sided signal and an early, left-right independent role for nodal flow
}

Jonathan Stevens ${ }^{1}$, Alexander Ermakov 1,4, Jose Braganca2,5,6, Helen Hilton'1, Peter Underhill1', Shoumo Bhattacharya², Nigel A Brown ${ }^{3}$ and Dominic P Norris*1

\begin{abstract}
Background: Vertebrates show clear asymmetry in left-right (L-R) patterning of their organs and associated vasculature. During mammalian development a cilia driven leftwards flow of liquid leads to the left-sided expression of Nodal, which in turn activates asymmetric expression of the transcription factor Pitx2. While Pitx2 asymmetry drives many aspects of asymmetric morphogenesis, it is clear from published data that additional asymmetrically expressed loci must exist.

Results: A L-R expression screen identified the cytoskeletally-associated gene, actin binding lim protein 1 (Ablim 1), as asymmetrically expressed in both the node and left lateral plate mesoderm (LPM). LPM expression closely mirrors that of Nodal. Significantly, Ablim 1 LPM asymmetry was detected in the absence of detectable Nodal. In the node, Ablim 1 was initially expressed symmetrically across the entire structure, resolving to give a peri-nodal ring at the headfold stage in a flow and Pkd2-dependent manner. The peri-nodal ring of Ablim 1 expression became asymmetric by the midheadfold stage, showing stronger right than left-sided expression. Node asymmetry became more apparent as development proceeded; expression retreated in an anticlockwise direction, disappearing first from the left anterior node. Indeed, at early somite stages Ablim 1 shows a unique asymmetric expression pattern, in the left lateral plate and to the right side of the node.

Conclusion: Left LPM Ablim 1 is expressed in the absence of detectable LPM Nodal, clearly revealing existence of a Pitx2 and Nodal-independent left-sided signal in mammals. At the node, a previously unrecognised action of early nodal flow and Pkd2 activity, within the pit of the node, influences gene expression in a symmetric manner. Subsequent Ablim 1 expression in the peri-nodal ring reveals a very early indication of L-R asymmetry. Ablim 1 expression analysis at the node acts as an indicator of nodal flow. Together these results make Ablim1 a candidate for controlling aspects of LR identity and patterning.
\end{abstract}

\section{Background}

While vertebrates are externally mirror symmetrical between their left and right sides, internally the positioning and patterning of their organs and vasculature show marked left-right (L-R) asymmetry. The heart and its associated vasculature, the lungs and various elements of the gut show distinct L-R asymmetric patterning. The

* Correspondence: d.norris@har.mrc.ac.uk

1 MRC Mammalian Genetics Unit, MRC Harwell, Harwell Science and Innovation Campus, Oxfordshire, OX11 ORD, UK

Full list of author information is available at the end of the article importance of correctly establishing L-R asymmetry is evident when the association between situs defects and disease is analysed. A strong association is evident with congenital heart disease [1] while links also exist with ciliary dyskinesia, cystic kidney disease and extrahepatic biliary atresia $[2,3]$.

During mammalian development, the first morphological sign of L-R asymmetry is the looping of the primitive heart tube, initially to the right. Shortly after this the embryo begins to undergo embryonic turning, the pro- 
cess that results in the embryo taking up the classic foetal position. This occurs in a L-R asymmetric manner such that the caudal-most region of the embryo passes to the right side of the head. These morphological asymmetries are, however, prefigured by molecular asymmetries. Work over the past decade has resulted in a broadly accepted model explaining how L-R asymmetry is established in the mammalian embryo (reviewed [4]).

Initial asymmetry is believed to be established when posteriorly tilted cilia within the embryonic node rotate to drive a leftwards flow of liquid (nodal flow). The role of flow in establishing situs was demonstrated in elegant experiments applying artificial flow to embryos in culture; flow reproducibly directed normally left-sided gene expression downstream of the direction of flow [5]. The question of how the embryo perceives nodal flow remains unresolved, although various models exist. One model argues that a morphogen is carried leftwards by the flow [6]. A second, the two cilia model [7], argues that mechanosensory cilia directly sense nodal flow, resulting in a left-sided intracellular calcium signal. The third model argues that membrane bound vesicles, termed nodal vesicular parcels (NVPs), are carried leftwards by the flow, breaking on the left side of the node to release a cargo of morphogens [8]. At present no one model fully explains all the existing experimental data [9]. The resulting signal at the left side of the node is then communicated several cell diameters to the left lateral plate, possibly through intracellular calcium signalling [7].

In the left lateral plate, the gene encoding the signalling molecule Nodal is asymmetrically activated downstream of nodal flow. Little is known of the mechanism of this activation. Nodal is at the top of a left-sided genetic cascade, auto-activating its own expression as well as that of its antagonist Lefty 2 and the downstream transcription factor Pitx2 (reviewed [4]). All are asymmetrically expressed prior to the appearance of morphological asymmetry [10]. While Nodal and Lefty 2 are expressed for only 6-8 hours [10], asymmetric Pitx2 expression is maintained into organogenesis and has been argued to be the ultimate effecter of left identity [11,12]. However, Pitx2 null embryos do not lose all aspects of left sidedness, making it clear that additional uncharacterised signals help distinguish the left and right sides of the early embryo [4].

We set out to identify additional asymmetric genes using a micro array based approach. This resulted in the identification of asymmetric expression of actin binding lim protein 1 (Ablim1), a gene showing asymmetry of expression in both the left LPM and the node. The lateral plate expression broadly mirrors that of Nodal, yet uniquely for a left LPM expressed locus, we demonstrate that it can be asymmetrically expressed in the absence of detectable LPM Nodal. This makes it clear that there is an additional uncharacterised asymmetric LPM signal. Initial Ablim1 expression in the ventral node was downregulated and peri-nodal expression upregulated in a flow and $P k d 2$-dependent manner, revealing an asymmetry independent role for flow in regulating gene expression within the node. The first node asymmetry was seen at the late headfold stage, earlier than any previously characterised L-R asymmetry in the mouse. Subsequently, the peri-nodal ring of expression retreated asymmetrically, moving around the node in a clockwise direction. Together these results reveal a Nodal-independent asymmetric LPM signal and make Ablim1 a candidate for controlling aspects of L-R identity and patterning.

\section{Results}

Ablim 1: a novel mammalian L-R asymmetric locus

To identify genes asymmetrically expressed between left and right sides in the developing embryo we compared gene expression using the MRC Mouse Known Gene Oligo Array printed array slides (Mm_SGC_Av2), which identify 7455 known loci. Left and right lateral plate tissue was dissected from 3-6 somite embryos and pools from 4 embryos were used to prepare RNA. Following SMART PCR amplification, hybridisation and analysis were performed as described in the Materials and Methods. The results from 4 replicates were analysed, and lists ranking the apparent degree of left or right sided asymmetry were generated (see Additional file 1). The presence, at the top of the left-sided list of the known left specific gene, Pitx2, demonstrated the validity of the approach. Significantly, Nodal, though present on the array, was not identified; Lefty 2 was not present on the array. We further analysed expression of 7 loci from the left-sided and 6 from the right sided list by RNA wholemount in situ hybridisation (WISH) on $8.5 \mathrm{dpc}$ embryos. Of the 13 loci examined, only one, Ablim1, showed apparent L-R asymmetry of expression (Fig. 1 and data not shown). Expression in the left LPM was clearly stronger and more extensive than in the right, while a second asymmetric domain was visible at the node. Initial analysis of a few embryos showed expression predominantly on the right hand side of the node.

Performing WISH using the full cDNA, we examined Ablim1 expression in a developmental series of embryos, from $6.5 \mathrm{dpc}$ to $9.5 \mathrm{dpc}$ (Fig. 1b-e). Expression was detected in the yolk sac of $6.5 \mathrm{dpc}$ embryos, initially as patches (Fig. 1b) that became a distinct ring of expression by $7.5 \mathrm{dpc}$ (Fig. 1c), consistent with it marking the prospective blood islands. At $7.5 \mathrm{dpc}$, expression was also seen in the developing head folds (Fig. 1c), an expression pattern maintained through $9.5 \mathrm{dpc}$ (Fig. 1d, e). Ablim 1 expression in the node was seen from $7.5 \mathrm{dpc}$ (Fig. 1c). By $8.5 \mathrm{dpc}$ L-R symmetric expression was evident in the developing heart. Strikingly, asymmetric expression was 


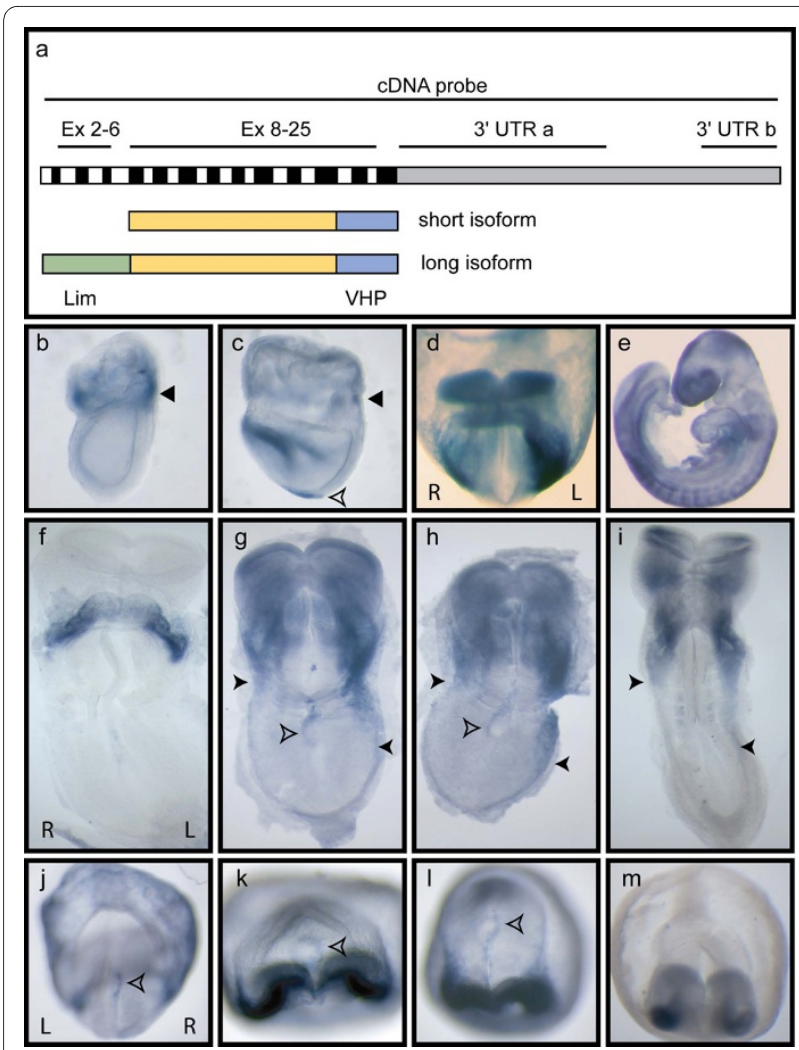

Figure 1 Ablim 1 is a novel asymmetrically expressed locus. (a) Diagram representing the Ablim 1 transcript. The relative positions of the probes used for WISH analysis are marked at the top of the panel. The exon structure is indicated in the middle and the two major protein isoforms of Ablim 1 are indicated at the bottom; the long isoform has 4 lim domains that the short form lacks. (b-m) Comparison of embryonic expression patterns of Ablim 1 using probes to different mRNA regions. (b-e) Developmental expression of Ablim 1 using the full length cDNA probe. (b) patchy yolk sac expression at $6.5 \mathrm{dpc}$, indicated by arrowhead, (c) a ring of expression in the yolk sac (arrowhead), head and node (open arrowhead) at $7.5 \mathrm{dpc}$ (d), symmetric expression in the head and yolk sac in addition to asymmetric expression is the lateral plate seen at $8.5 \mathrm{dpc}$, (e) expression in the heart, head and somites at $9.5 \mathrm{dpc}$. Expression at $8.5 \mathrm{dpc}$ was assessed with Ex2-6 (f, j), Ex8-25 (g, k), 3'UTRa ( $h, I)$ and 3'UTRb (l, m). (f-i) flat mounted 8.5 dpc embryos dissected from their yolk sacs, visualised ventrally. (j-m) $8.5 \mathrm{dpc}$ embryos within yolk sacs visualised from the posterior. The posterior most extent of LPM expression is indicated by a closed arrowhead and node expression is indicated by an open arrowhead. Panels (b), (c) and (e) are lateral views. In other panels Left (L) and Right (R) are as marked and are the same for each row of panels.

also seen in the LPM; stronger and more extensive on the left than the right (Fig. 1d). By $9.5 \mathrm{dpc}$ expression was evident in the head, the somites and portions of the heart, but no L-R asymmetry was seen at this stage (Fig. 1e, data not shown).

\section{Two classes of Ablim 1 transcript show asymmetric} expression in the lateral plate and the node respectively Analysis of the published data [13] and ESTs http:// www.ensembl.org demonstrates the existence of multiple
Ablim1 transcripts, exhibiting both alternative splicing and multiple alternative first exons. Northern blot and RT-PCR analysis confirmed the existence of Ablim1 transcripts in 7.5 and $8.5 \mathrm{dpc}$ embryos (data not shown). While multiple protein isoforms of Ablim1 exist, they comprise two major classes; long forms containing lim domains and a villin head piece (VHP) and a short form lacking the lim domains [13] (Fig. 1a). We investigated the temporospatial distribution of the transcripts encoding these isoforms using WISH probes hybridising to different portions of the Ablim1 message; probes corresponding to the beginning (Ex 2-6), the common region (Ex 8-25) and the 3'UTR (3'UTRa and b; Fig. 1). The Ex 2-6 probe, encompassing the lim domains, revealed expression in the developing heart (Fig. 1f) as well as the right side of the node when WISH colour development was extended for longer times (Fig. 1j), but not in the lateral plate. The Ex 8-25 probe revealed asymmetric expression to the right side of the node and in the left lateral plate; symmetric expression was evident in the head and heart (Fig. 1g, k). The 3'UTRa probe showed similar expression to Ex8-25, although node expression was significantly weaker when compared to the other expression domains (Fig. 1h, l). The terminal 3'UTR probe, 3 'UTRb, showed similar expression, but failed to detect node expression, arguing for a shorter $3^{\prime} U T R$ in the node transcripts (Fig. 1i, m). Together these results argue that a transcript encoding a long isoform containing both lim domains and VHP is present in the node, while a shorter isoform (with no lim domains) is present in the left lateral plate; it is not possible to say from our WISH or RT-PCR analysis whether the short isoform is also present in the node.

\section{Asymmetric Ablim 1 lateral plate expression mirrors Nodal}

Nodal, often thought of as the master gene controlling left-sided gene expression, is expressed in the left but not right lateral plate from 3-6 somite stages [14,15]. If asymmetric Ablim1 expression is directly controlled by Nodal, it would be expected to exhibit similar temporospatial expression and not be expressed asymmetrically prior to asymmetric Nodal expression. We therefore examined the temporospatial expression of Ablim1 from the late headfold to the 10 somite stage by WISH (using the 3'UTRa probe). Up to and including the 2 somite stage, bilaterally symmetrical expression was seen in the anterior lateral plate, contiguous with expression in the heart (Fig. 2a). By 3 somites expression in the LPM became clearly asymmetric, with expression in the left lateral plate being both stronger and stretching noticeably further posteriorly than in the right (Fig. 2b). This asymmetric expression was highly evident at 5 somites, being particularly obvious when the WISH is developed for a short time (Fig. 2c). By 7 somites, after lateral plate Nodal expression has ceased, asymmetric left lateral plate 


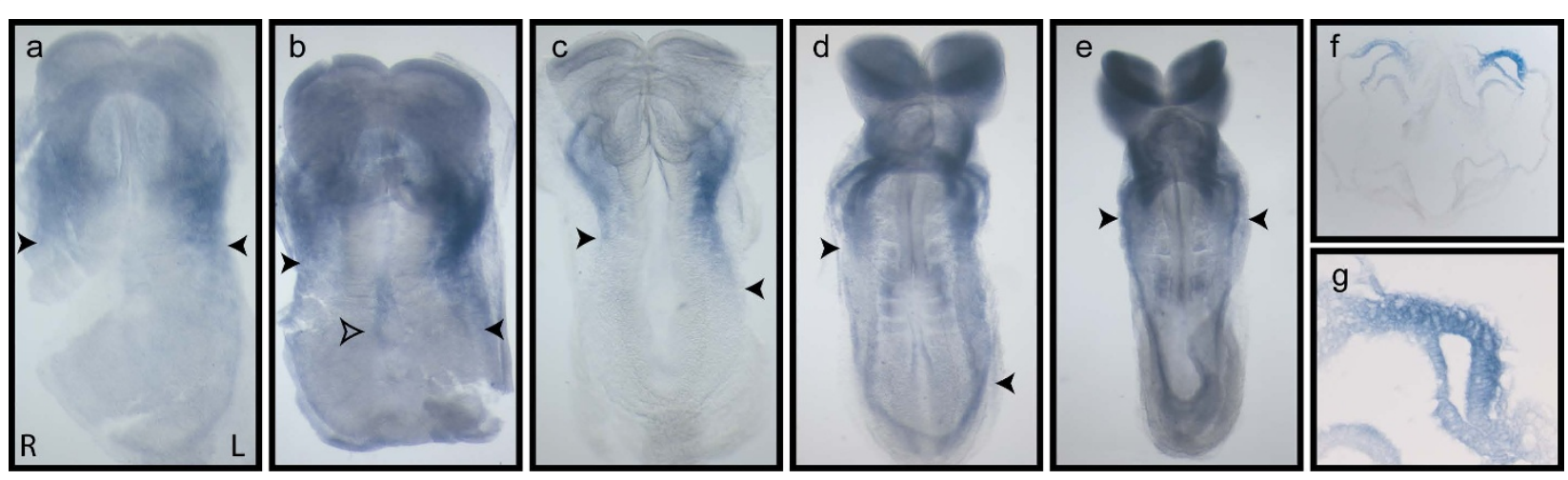

Figure 2 Ablim 1 lateral plate asymmetry mirrors Nodal. Ablim 1 is bilaterally symmetrical at 2 somites (a), becoming obviously asymmetric by 3 somites (b), when left lateral plate expression is stronger and more extensive than on the right. This WISH has been developed for longer allowing the asymmetric node expression to be visualised (open arrowhead). LPM asymmetry remains obvious at 5 somites (c) and some left LPM asymmetry is visible at 7 somites. Symmetrical expression in the more posterior somites is also evident. By 8 somites, all L-R asymmetry has been lost (e). Histology shows that Ablim 1 is expressed throughout the lateral plate mesoderm $(f, g)$. The posterior most extent of LPM expression is indicated by a closed arrowhead. In all panels the 3'UTRa probe has been used.

expression of Ablim1 was strongly downregulated, although still evident at a low level in some embryos (Fig. 2d). By 8 somites, no sign of asymmetric lateral plate expression was evident (Fig. 2e). Analysis of sections revealed that lateral plate expression was present throughout the lateral plate mesoderm (Fig. 2f, g) similar to Nodal. These data are consistent with the hypothesis that Nodal activates Ablim1. As with our previous results, expression of Ablim1 in the node was apparent when the WISH colour development was left for longer periods of time (Fig. 1h, l, 2b).

\section{Left lateral plate Ablim1 expression occurs in the absence of detectable LPM Nodal}

To further test the hypothesis that Nodal activates asymmetric Ablim1 expression, Ablim 1 lateral plate expression was analysed in mutants with abnormal L-R patterning. Dnahc11 encodes a dynein heavy chain required for nodal cilia motility: the point mutant Dnahc11 iv (iv) results in immotile nodal cilia, absence of nodal flow, and randomisation of both situs and Nodal lateral plate expression [15-18]. When Ablim1 lateral plate expression was analysed in $i v$ mutants, a mixture of expression patterns were seen (Table 1), similar to those previously reported for Nodal in the iv mutant [15], including leftsided (Fig. 3a), bilateral (Fig. 3b) and right sided (Fig. 3c) expression. These data show that Ablim1 asymmetry is downstream of nodal flow, similar to Nodal asymmetry and is consistent with Ablim1 asymmetry being downstream of Nodal. This hypothesis was further supported by analysis of Shh mutant embryos; Shh mutants do not express the Nodal antagonist Lefty1 in the midline, resulting in bilateral Nodal expression [19]. Consistent with a role for Nodal upstream of lateral plate Ablim1 expres- sion, bilateral Ablim1 expression was seen in $S h h^{-1-}$ embryos (data not shown and Table 1).

We sought to analyse Ablim1 expression in embryos where Nodal LPM expression was strongly downregulated. Nodal contains an intronic enhancer (ASE) that is responsible for positive feedback regulation of its asymmetric expression in the left LPM. The NodalD600 allele lacks this ASE and consequently NodalD600/D600 embryos express extremely low levels of Nodal in the LPM $[20,21]$. This results in delayed and posteriorised activation of the Nodal target Pitx2, leading to L-R patterning defects. When Ablim1 expression was analysed in NodalD600/D600 embryos, 50\% of embryos showed asymmetric expression in left LPM (Fig. 3d; Table 1); the remainder were symmetrical, showing no LPM expression. However, the frequency at which no lateral plate Ablim1 expression was detected in NodalD600/D600 embryos was far higher than in wild type (Table 1). It is possible that the change in Ablim1 expression is a consequence of the delayed activation of the transcription factor Pitx2 in the NodalD600/D600 embryos. Therefore, we analyzed Ablim1 expression in Pitx2 mutants; Pitx2c is the isoform expressed asymmetrically in the left lateral plate mesoderm and Pitx2c-/- embryos show defects in L-R patterning [22]. All 5 Pitx2c-/- embryos analysed showed wild type Ablim1 expression (Table 1; Fig. 3e) demonstrating that Pitx2c expression is not required for Ablim1 asymmetry.

It seemed possible that the low level of LPM Nodal expressed in Nodal ${ }^{D 600 / D 600}$ embryos is borderline for activating asymmetric expression of Ablim1 in the left LPM and therefore leads to stochastic activation of the locus. To determine whether the $50 \%$ of NodalD600/D600 embryos that expressed asymmetric LPM Ablim1 
Table 1: LPM Ablim 1 expression.

\begin{tabular}{|c|c|c|c|c|}
\hline Embryo & $\begin{array}{l}\text { Left } \\
\text { LPM }\end{array}$ & $\begin{array}{l}\text { Left and Right } \\
\text { LPM }\end{array}$ & $\begin{array}{l}\text { Right } \\
\text { LPM }\end{array}$ & $\begin{array}{l}\text { No LPM } \\
\text { Expression }\end{array}$ \\
\hline Wild Type & $61(80 \%)$ & & & $15(20 \%)$ \\
\hline iv/iv & $2(11 \%)$ & $8(44 \%)$ & $5(28 \%)$ & $3(17 \%)$ \\
\hline NodalD600/D600 & $5(56 \%)$ & & & $4(44 \%)$ \\
\hline Nodalnode/- & $6(24 \%)$ & & & $19(76 \%)$ \\
\hline Pitx2c-/- & $5(100 \%)$ & & & \\
\hline Shh-/- & & $4(100 \%)$ & & \\
\hline
\end{tabular}

resulted from low level Nodal expression, we analysed lateral plate Ablim1 expression in Nodal ${ }^{\Delta n o d e /-}$ embryos. The Nodal ${ }^{\Delta n o d e}$ allele lacks the enhancer that drives Nodal

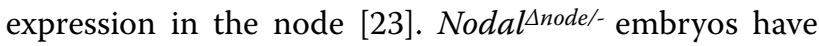
no, or occassionally a minimal ammount of Nodal expression at the node and no detectable Nodal, Lefty 2 or Pitx 2 in the LPM [23]. When Ablim1 expression was analysed in Nodal ${ }^{\text {nnode/- }}$ mutant embryos, of 25 analysed, 6 (24\%) showed clear asymmetric left LPM expression (Table 1, Fig. 3g). The remaining 19 (76\%) showed no LPM Ablim1 expression on either the right or left sides (Table 1, Fig. 3f). These data clearly demonstrate that Ablim1 can be asymmetrically expressed in the LPM in the absence of

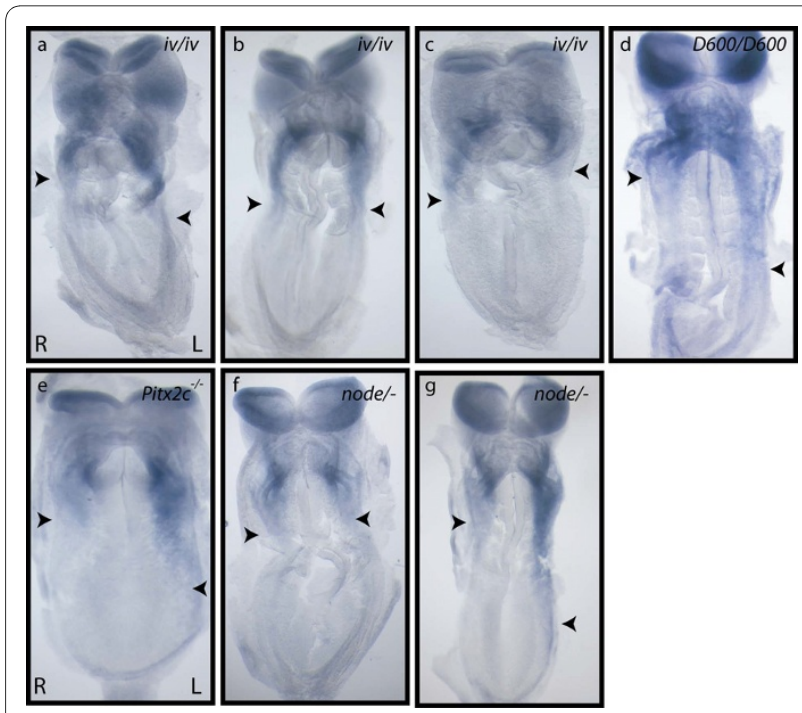

Figure 3 Ablim 1 lateral plate asymmetry is maintained in the absence of Nodal. Expression, as assessed by WISH with the 3'UTRa probe. In iv/iv mutants (a-c), (a) left-sided, (b) bilateral and (c) right sided expression was detected. (d) Left-sided Ablim 1 expression in a NodalD600/D600 (D600/D600) embryo. (e) Normal, left-sided expression of

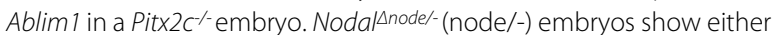
loss of asymmetric Ablim 1 expression (f) or left-sided Ablim 1 expression (g). The posterior most extent of LPM expression is indicated by a closed arrowhead. detectable LPM Nodal signalling and therefore suggests that this asymmetric expression is regulated by signalling cues that are independent of the Nodal cascade. The reduced frequency of asymmetric Ablim1 expression in Nodal ${ }^{\text {nnode/- }}$ embryos, however, suggests a role for Nodal in the robustness of Ablim1 lateral plate asymmetry. When we re-analysed these data with respect to the stage of development (Table 2), we saw no asymmetric LPM Ablim 1 expression before 4 somites. Approximately $30 \%$ of 4-5 somite embryos showed asymmetric LPM Ablim1 expression. This increased to $50 \%$ when the $6-7$ somite embryos were analysed. Together these data demonstrate that detectable LPM Nodal is required for early asymmetric left LPM Ablim1 expression, but, that in its absence a second asymmetric system can activate Ablim1 asymmetry.

\section{Ablim 1 shows no functional ASE}

In the lateral plate, Nodal's auto-activation of its own expression, as well as its activation of Lefty 2 and Pitx 2 expression, is mediated by binding of FoxH1 to asymmetric elements (ASEs). ASEs contain two or three FoxH1 binding sites (TGT G/T T/G ATT) within a 30-200 bp region. The random frequency of a pair of binding sites within such a region is once every $350 \mathrm{~kb}$. When ASE-like sequences were sought around the mouse and human Ablim1 loci, 7 sequences were identified in mouse and 3 in humans (see Additional file 2), although position and overall sequence was not conserved. To address whether Nodal might be interacting with Ablim1 through these elements, the sequences plus $100 \mathrm{bp}$ on either side were PCR amplified and cloned into luciferase reporter vectors. These were transfected into HepG2 cells together with $F o x H 1$ and a constitutively active Alk4 construct. While a control fragment from the mouse Pitx2 ASE activated luciferase, as previously reported [24], all the Ablim1 derived fragments failed to activate expression above background levels, arguing that Nodal does not activate Ablim1 through FoxH1 binding to an ASE (data not shown). 
Table 2: LPM Ablim 1 expression in Noda/node/null mutant embryos classified by somite stage*

\begin{tabular}{lll}
\hline Nodalnode/null & Asymmetry & No Asymmetry \\
\hline 2-3 Somites & & \\
4-5 Somites & $0(0 \%)$ & $9(100 \%)$ \\
$6-7$ Somites & $3(30 \%)$ & $7(70 \%)$ \\
\hline
\end{tabular}

${ }^{*}$ (these are the same embryos analysed in Table 1)

\section{Highly dynamic Ablim 1 node expression: a very early marker of asymmetry}

When temporospatial Ablim1 expression was analysed in the node, utilising the Ex2-6 probe, transcript was detected from when the node is first patent (Fig. 4a, b). Intriguingly, this first expression is of a salt and pepper pattern stretching across the pit of the node. This resolved to give a ring surrounding the node by the midheadfold stage (Fig. 4c); the first indication of asymmetry was evident at this stage, with a higher level of expression on the right than the left hand side of the node (Fig. 4c). During the next few hours of development, expression on the anterior left side of the node was lost, while expression was activated in the midline cells anterior to the node, resulting in a question mark-like expression pattern (Fig. 4d). By 4 somites, the remaining left-sided expression was lost, resulting in solely right-sided expression at the node (Fig. 4e). This abrogation of expression continued in a clockwise direction around the node (Fig. 4f), until by 7 somites peri-nodal expression was restricted to midline cells anterior to the node (Fig. 4g). By 8 somites midline expression has also been lost (Fig. 4h). Sections through these embryos reveal that expression in the node is restricted to the ventral layer (Fig. 4i).

\section{Ablim 1 node expression is controlled by nodal flow and Pkd2 activity}

The early dynamic expression pattern of Ablim1 in the node correlates with the changes in nodal cilia motility and nodal flow described by Okada [18]. The loss of Ablim1 expression from the pit corresponds to the stage when local vortices form, while the loss of expression from the left side of the node correlates with the establishment of a strong leftwards flow. In conjunction with the very early asymmetry of Ablim1, this suggests that expression may be responding directly to nodal flow. To test this hypothesis we next examined Ablim1 node expression in iv mutants, where there is no nodal flow. Of 40 mutant embryos analysed, no asymmetry was detected at any stage of development. Indeed, the robust peri-nodal ring of expression was never detected. There was, however, a rise in the number of embryos where expression was not detected, from $12 \%$ in wt to $42 \%$ in $i v /$ iv mutants (Table 3). Intriguingly, in the $58 \%$ of embryos where expression was detected, the pattern of expression was the same patchy expression seen in the very earliest wild type nodes (Fig. 5a). This pattern was maintained well through the period that leftwards laminar flow is normally detected and that strong asymmetry of Ablim1 is normally seen at the node. These data demonstrate that nodal flow is required for the upregulation of Ablim1 expression in the peri-nodal region and is involved in downregulation of Ablim1 expression in the pit of the node.

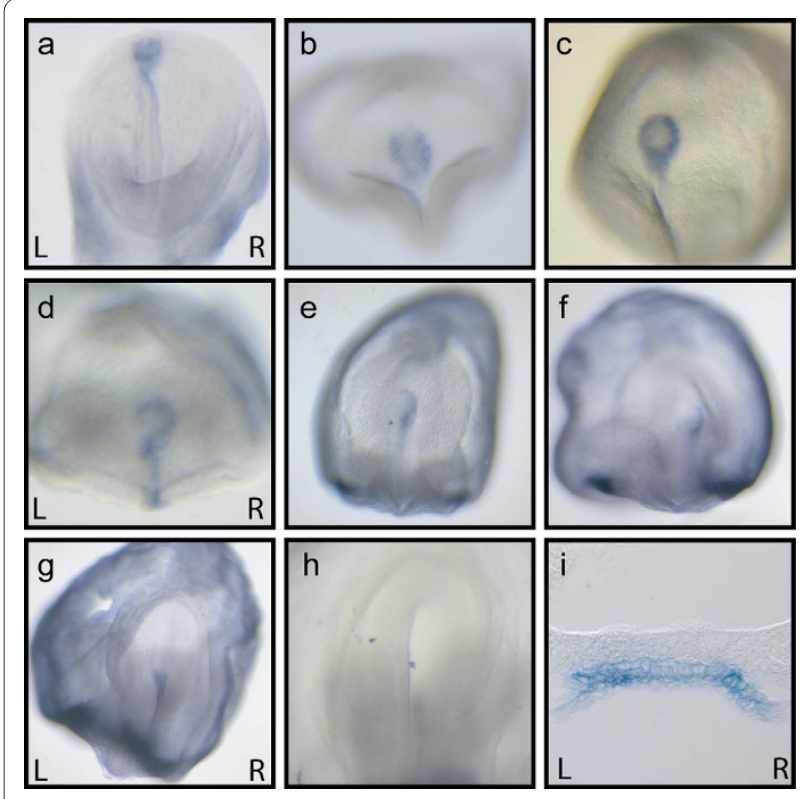

Figure 4 A novel asymmetric expression pattern for Ablim 1 at the node. Temporal analysis of Ablim 1 expression in the node, using the Ex2-6 probe. Initial expression across the node at the Early Headfold stage (a), becomes modified by the mid headfold stage (b) and by late headfold stages (c) describes a peri-nodal ring that shows stronger right than left-sided expression. By early somite stages (d) peri-nodal expression has been lost from the anteriormost left side of the node as well as being evident in the midline. (e) By 4 somites expression is restricted to the right side of the node. (f) At 5 somites node expression is restricted to the anteriormost right side and becomes restricted to the midline by 7 somites (g). (h) By 8 somites expression was absent form the node. (i) A section of a late headfold embryo (as in panel c) showing that expression in the node is restricted to the ventral layer of the node. Left $(\mathrm{L})$ and Right $(\mathrm{R})$ are as indicated on the panels. 
Table 3: Node Ablim 1 expression.

\begin{tabular}{llll}
\hline Embryo & $\begin{array}{l}\text { Normal Node } \\
\text { Expression }\end{array}$ & $\begin{array}{l}\text { No Detectable Node } \\
\text { Expression }\end{array}$ & $\begin{array}{l}\text { Patchy" Node } \\
\text { Expression }\end{array}$ \\
\hline Wild Type & $37(88 \%)$ & $5(12 \%)$ & $23(58 \%)$ \\
iv/iv & & $17(42 \%)$ & $5(56 \%)$ \\
Pkd2/LRM4 & $4(44 \%)$ & \\
\hline
\end{tabular}

The two cilia hypothesis argues that nodal flow is directly detected by nodal cilia, through the activity of $\mathrm{Pkd} 2$ [7], resulting in a left-sided $\mathrm{Ca} 2+$ signal. To test whether Ablim 1 may be responding to these asymmetric $\mathrm{Ca} 2+$ signals, we analysed expression in $\mathrm{Pkd} 2$ mutants. Surprisingly, very similar result were obtained to those for the iv mutant. From 9 mutants analysed, 4 (44\%) showed no detectable expression, while 5 showed the same patchy expression we detected in the embryos lacking nodal flow (Fig. 5b). Once again, no L-R asymmetry was detectable. Together, these data demonstrate a requirement for both nodal flow and $\mathrm{Pkd} 2$ within the pit of the node for the loss of Ablim1 expression as well as for the establishment of asymmetric expression surrounding the node.

\section{Discussion}

In this paper we describe the novel L-R asymmetric expression pattern of Ablim1, a gene that can be expressed independently of detectable Nodal in the left lateral plate mesoderm. A separate asymmetric expression domain in the embryonic node reveals both that Ablim1 expression is the earliest known marker of mammalian L-R asymmetry, and that flow (most likely mediated through $\mathrm{Pkd} 2$-dependent $\mathrm{Ca} 2+$ signalling) is directly affecting gene expression in the node, prior to any effects on asymmetry.

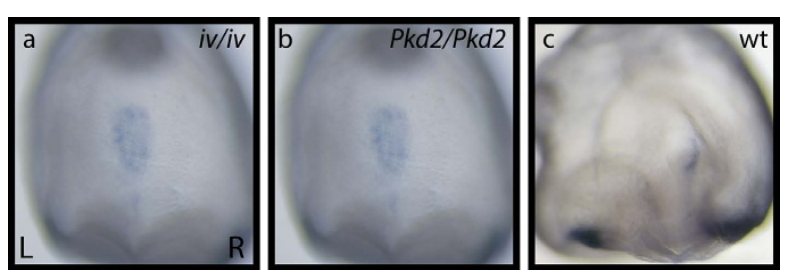

Figure 5 Nodal flow and $P$ kd 2 control Ablim 1 expression at the node. (a) Ablim 1 expression in a 4 somite Pkd2 mutant. (b) Ablim 1 expression in a 5 somite iv mutant. (c) Ablim 1 expression in a 5 somite wild type control embryo. In both iv mutants, which lack nodal flow and $P k d 2$ mutants, Ablim 1 expression was not detected in the usual peri-nodal ring; a patchy expression across the floor of the node was seen in a significant proportion of embryos, in striking contrast to wild type control embryos (c)
A Nodal independent L-R asymmetric signal ("signal X")

Work from many research groups over the past decade has established a generally accepted pathway underlying establishment of L-R patterning in mammals (reviewed [4]). The activation of the Nodal signalling cascade in the left LPM results in asymmetric, left-sided, expression of Pitx2 that is maintained into organogenesis (Fig. 6). In light of misexpression experiments in chick and Xenopus, Pitx 2 has been argued to specify left sidedness $[11,12]$. Yet, while Pitx 2 mutant mouse embryos demonstrate right pulmonary and atrial isomerism [25-28], the initial direction of embryonic turning and heart looping are Pitx2-independent [22]. In contrast, analysis of mice lacking or unable to respond to Nodal signalling in the lateral plate showed a randomised direction of heart looping $[23,29]$; the direction of embryonic turning was also randomised in Cryptic (MGI: Cfc1) mutants, but not reported for the Nodal mutants [23,29]. These data argue that heart looping and embryonic turning are controlled by Nodal expression, however, it is not possible to distinguish the role of Nodal at the node from its role in the lateral plate in these experiments. Our results clearly show Ablim1 asymmetry is independent of Pitx 2 expression. More significantly, Ablim1 is capable of being asymmetrically expressed in the absence of detectable LPM Nodal signalling. Quite clearly, another L-R asymmetric signal is present in the embryo, that for the sake of discussion we shall refer to as "signal X".

\section{The control of Ablim1 LPM asymmetry}

While it is clear from our analysis that Ablim1 can be asymmetrically expressed in the absence of detectable Nodal and Pitx2, asymmetric Nodal obviously does play a role. The strong reduction of lateral plate Nodal expression in Nodal ${ }^{D 00 / D 600}$ embryos results in $\sim 50 \%$ of embryos with detectable asymmetric LPM Ablim1 expression, while the total removal of LPM Nodal expression in the Nodal ${ }^{\Delta \text { node/- }}$ embryos reduces this level to $24 \%$ (Table 1). The level of LPM Nodal expression therefore directly affects asymmetric Ablim 1 expression. This relationship is underlined when the temporal activation of Ablim1 is analysed; strong reduction of Nodal signalling 


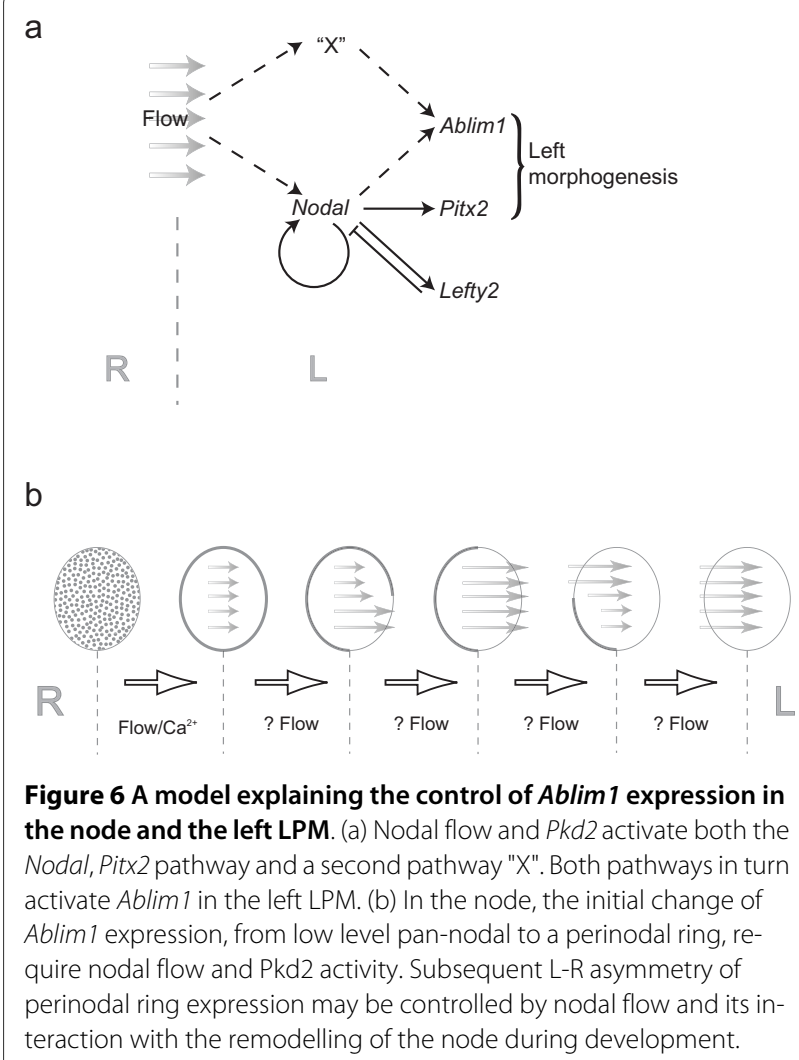

is known to result in delayed target gene activation [20]. At early somite stages no Nodal-independent Ablim1 expression is seen, compared to expression in $30 \%$ of embryos by $4-5$ somites and $50 \%$ by 6-7 somites (Table 2 ). It is therefore clear that two signals, a Nodal-dependent and the Nodal-independent "signal X" influence asymmetric lateral plate Ablim1 (Fig. 6A). While we have drawn "signal X" and Nodal both directly acting on Ablim1, we cannot rule out the possibility that "signal X" is in part regulated by Nodal. It is also possible that there is a temporal offset between Nodal signalling and "signal X", with Nodal acting first. The data neither argue for nor against these scenarios and in the absence of identifying and interfering with "signal $\mathrm{X}$ " it is impossible to distinguish between them.

\section{A left-sided "signal X"}

A signal acting on the left side of the embryo may reflect either a left-sided activator or a right sided repressor of Ablim1 expression. Work from various groups has revealed additional lateral plate asymmetries in gene expression. BMP signalling, as assessed by Smad1 phosphorylation, is L-R asymmetric and this asymmetry is driven by asymmetric expression of the BMP antagonists chordin and noggin, which in turn are controlled by Nodal [30]. Therefore, it seems unlikely that BMP signalling lies upstream of Ablim1 asymmetry. Similarly, the
Nkx3-2 (or BapX1) homeodomain locus shows right sided asymmetric expression, but again is thought to act downstream of Nodal [31]. In contrast, little is known about control of the right sided transcription factor Snail (Snai1), which temporally mirrors Nodal [32]. Conditional deletion leads to randomised embryonic turning and heart looping and bilateral activation of the Nodal signalling cascade, arguing that it normally inhibits right sided Nodal expression. It is therefore possible that Snai1 inhibits Ablim1 expression on the right side of the embryo. Indeed the role of the snail family of genes as transcriptional repressors is well documented [33]. A formal possibility exists that "signal $\mathrm{X}$ " in fact represents a very low level Nodal signal, below that capable of activating the known LPM targets of Nodal. While no Nodal expression is detected in the LPM, low level ectopic Nodal expression was reported in a few cells in the pit of the node in a small proportion of Nodal ${ }^{\Delta n o d e /-}$ embryos [23]. Such a localised signal would be expected to first activate expression close to the node, in a manner similar to that reported for initial Nodal activation [discussed in [4]. No such localised expression was evident for Ablim1 in Nodal ${ }^{\Delta n o d e /-}$ embryos (Fig. 3g and data not shown). Indeed, such putative low level Nodal signalling falls outside of the known activity of Nodal and it too would arguably constitue a novel asymmetric signal.

\section{Flow regulates early symmetrical Ablim 1 expression at the node}

Wild type Ablim1 expression in the node changes markedly at the mid-late headfold stage, from a low level broad pan-node expression to a robust peri-nodal ring (Fig. 4). This reflects two changes; upregulation in the crown cells surrounding the node and downregulation within the pit of the node. Developmentally this corresponds to when nodal cilia are driving vortical fluid motion [18], suggesting that cilia driven fluid flow plays a role in these expression changes. This is supported by the failure of $i v$ and $P k d 2$ mutant embryos to express the peri-nodal ring of Ablim1 expression (Table 3). Moreover, almost $60 \%$ of these embryos maintain the same low level pan-node expression seen in early wild type embryos. We detected no expression in nodes of the remaining mutant embryos and argue that this reflects either failure to maintain low level pan-node expression over time and/or technical limitations in detecting low level gene expression by WISH. The role of nodal cilia motility in generating nodal flow and L-R asymmetry is so central to thinking that little consideration has been given to any earlier role for fluid flow. Our data, however, reveals an early and previously unrecognised function for nodal flow in modulating symmetrical gene expression within the node, separate from the role of nodal flow in L-R determination. 


\section{Ablim 1 is the earliest marker of L-R asymmetry in mouse} The first L- $\mathrm{R}$ asymmetry in Ablim1 expression is evident in the node by the late headfold stage (Fig. 4c), several hours before the 1-2 somite stage at which asymmetry is evident for the other known asymmetric loci Nodal, Cerl2 (MGI: Dand5) and Lplunc1 [14,15,34-36]. This argues that Ablim1 is responding to very early asymmetric signals. Nodal flow is argued to be the initial asymmetric signal in the mouse (reviewed [4]) and is clearly driving fluid flow leftwards by early somite stages [18]. However, the first Ablim1 asymmetry is evident at a stage when beads introduced into a node in vitro are carried leftwards only inefficiently and on average, hopping from vortex to vortex [18]. Whether such an inefficient flow could affect sensory cilia sufficiently to fulfil the requirements of the two cilia hypothesis is unclear. Presumably NVPs could be carried leftwards in a similar manner to the beads, although whether they would break efficiently in such a flow is uncertain.

By the early somite stages a completely novel and very distinctive asymmetry of Ablim1 expression becomes evident at the node. The peri-nodal ring "retreats" around the node in a clockwise direction between 3 and 7 somites. This asymmetry of expression is very different from that seen for other asymmetrically expressed loci at the node; these show bi-lateral expression flanking the node, with stronger expression on one side than the other.

While the control of Ablim1 node asymmetry is not addressed by this study, the speed of the changes in Ablim1 expression suggests that this is an active process. In chick an anti-clockwise migration of cells around the node underlies gene asymmetry $[37,38]$. However, in mice, cre-loxP based lineage analyses of both node crown and pit cells did not reveal such cell migration [23,39]. The role of flow in controlling earlier changes in Ablim1 expression makes flow a mechanism we must contemplate. Yet for flow to control Ablim1 asymmetry, the following objections must be taken into account. (1) How could leftwards flow initially affect just the anterior node? At early somite stages the anterior node is shallower than the posterior $[40,41]$ and this may influence the ability of flow to impact on the crown cells in the anterior versus the posterior. (2) How does flow subsequently affect the posterior node? As the embryo grows the node remodels, becoming more even in depth between anterior and posterior. At the same time leftwards flow becomes laminar. A combination of these two events may then allow flow to also affect posterior left-sided node crown cells. (3) How does flow subsequently affect the right side of the node? In vivo, fluid flow within the node recycles being drawn downwards on the right hand side [42]. A combination of growth, node remodelling and perhaps temporal accumulation of signalling may allow the right side of the node to respond to the recycled flow as it is pulled back into the right hand side of the node (Fig. 6B).

While there is strong evidence for nodal flow in many vertebrates [43-45], earlier, pre-flow events have been demonstrated to influence L-R patterning in non-mammalian species. Vg1 can influence situs determination and its putative co-receptor, Syndecan-2, becomes asymmetrically phosphorylated in pre-flow Xenopus embryos [46-49]. Pharmacolgical experiments have implictaed $\mathrm{H}^{+} \mathrm{K}+$ ATPase, VATPase, Serotonin and 14-3-3 family member $E$ in Xenopus situs determination [50-52]. In the resulting serotonin model, an electric field drives serotonin through gap junctions in Xenopus, resulting in higher right than left sided localisation [52-54]. The $\mathrm{H}^{+} \mathrm{K}^{+}$ATPase mRNA similarly becomes asymmetrically localised in Xenopus and perturbed expression disrupts L-R patterning in both Xenopus and chick [52]. Therefore the question must be raised as to whether such early mechanisms also exist in mammals and may be controlling Ablim1 asymmetry, either at the node or in the LPM.

That there is asymmetry of Ablim1 expression at both the node and the LPM bears comparison to the expression pattern of Nodal. Nodal asymmetry at the node slightly predates that in the LPM and is required for LPM expression in the mouse [23]. It has even been argued, in light of the ability of Nodal to autoactivate, that Nodal at the node might be carried to left LPM to activate expression there [55]. In striking contrast, Ablim1 asymmetry is on opposite sides in the node and LPM. So while there is Ablim 1 asymmetry at the node when LPM asymmetry is first detected, the expression domains are on opposite sides of the embryo (Fig. 1g). Moreover, while Nodal is a signalling molecule, Ablim1 is a structural, cell autonomously acting protein. It is difficult to envisage how a cytoskeletal protein would be acting to repress its own expression across many cell diameters. More likely, the two expression domains are independently regulated. Indeed, the long isoform of the protein seen at the node but not detected in the LPM originates at an alternate first exon, consistent with different promoter enhancer combinations controlling the two expression domains.

\section{Ablim1 Function}

Uniquely, for mammalian asymmetric genes, Ablim1 encodes a structural protein. As its name suggests, Ablim 1 protein binds to actin and when first identified, the presence of lim domains led the authors to suggest that it might act as an adaptor protein, bringing other proteins to the actin cytoskeleton [56]. The homologue in C. elegans, unc-115, similarly binds actin [57], and when mutated leads to an uncoordinated phenotype and defects in axon guidance [58]. Expression of a dominant negative Ablim1 in chick embryos leads to similar axonal phenotypes [59]. Yang and Lundquist [60] further dem- 
onstrated that expression of unc-115 in mammalian fibroblasts led to the formation of peripheral actin conglomerations at the expense of stress fibres. One possible explanation that they suggest is that Ablim1 protein may have different roles at the cell membrane and in the cytoplasm, acting to abrogate stress fibre formation when cytoplasmic. This raises the possibility that asymmetric Ablim1 expression might prove permissive for asymmetric morphogenetic changes in the embryo. However, when an isoform specific deletion of Ablim1 was made, for the isoform seen in the eye, no defects were reported [13]. This deletion, however, seems unlikely to affect the expression that we have described. Indeed many additional alternative first exons are now annotated that were not evident to $\mathrm{Lu}$ and colleagues.

\section{Conclusion}

We have identified Ablim1 as a L-R asymmetrically expressed LPM gene that also shows a highly novel asymmetric expression pattern in the node. Through study of Ablim1, we provide definitive evidence that in addition to the recognised Nodal-Pitx2 asymmetric pathway in the left LPM, a second LPM Nodal-independent pathway must exist.

In the node we reveal a previously unrealised role for flow and $P k d 2$ in the control of early symmetrical Ablim 1 expression within the node. This provides a novel, expression based readout of nodal flow. It seems reasonable to speculate that other genes expressed within the node may also be modulated by fluid flow.

Ablim1 expression within the early node becomes asymmetric at the head fold stage, several hours previous to other asymmetrically expressed loci. This is the earliest marker of L-R asymmetry in the mouse. Subsequent asymmetric node expression proceeds in an entirely novel pattern, retreating around the node. While we do not understand how this is controlled, future study of this seems likely to shed light on the mechanisms of L-R patterning.

Ablim1 is a candidate for processes controlling L-R morphogenesis and identity and future study of mutants will reveal it role in L-R patterning.

\section{Methods}

Mice

To collect staged embryos, mating was assessed by monitoring for copulation plugs. The day of plug was designated day 0.5 . Wild type embryos were $(\mathrm{C} 3 \mathrm{H} / \mathrm{HeH} \times 101 /$ H)F1s. Mutant lines were: $i v-D n a h c 11^{i v}[61,62] ; P k d 2^{l r m} 4$ [63]; Nodal ${ }^{\mathrm{D600}}$, Nodaltm2Rob [20]; Nodal is the NodalLacZ allele Nodal tmiRob [14]; Nodal ${ }^{\Delta n o d e}$ is the Nodal node

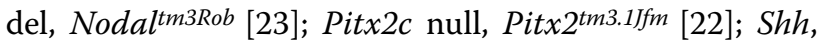
Shh ${ }^{\text {tm1Chg }}$ [64].
All animals were used in accordance with UK Home Office regulations.

\section{Microarrays}

Tissue for micro-array analysis was dissected in cooled PBS, then snap frozen in liquid nitrogen. RNA was produced by Qiagen RNeasy mini kit and quality assessed by Agilent 2100 Bioanalyzer. Reverse transcription and amplification were conducted according to the SMART mRNA Amplification Kit (Clontech). The resulting samples were labelled with $\mathrm{Cy} 3$ and $\mathrm{Cy} 5$ and used to hybridise MRC Mouse Known Gene Oligo Array printed array slides (Mm_SGC_Av2), identifying 7455 known genes, according to standard protocols. Results from 4 experimental repeats were analysed using GeneSpring (Agilent Technologies). Genes were ranked for differences in left versus right sided expression. The data from these experiments has been submitted to ArrayExpress, reference EMEXP-2277.

\section{Molecular biology}

The following IMAGE clones (IC) were used to produce anti-sense WISH probes: IC1-3995027 Oaz1, IC24457726 Agtrl1, IC3-4935411 Ablim1, IC4-5042041 Tssc3, IC5-5123607 F10, IC6-5291579 Fkbpla, IC75329923 Cts1, IC8-5716506 Plk, IC9-6335891 Hmgi, IC10-6390454 Aqp3, IC11-3963483 Tpm1, IC12-5715646 tuba1, IC13-5685601 Hyal1.

IC3 represents a full length Ablim1 clone. Clones portions of Ablim1 sequence, exons 2-6, 8-25 and 3'UTR sequences, were produced by restriction digest or PCR amplification and cloned into pBluescript2. Antisense RNA probes were produced and used for wholemount in situ hybridisation, according to standard protocols.

Putative ASE sequences were amplified by PCR from $\mathrm{BALB} / \mathrm{c}$ mouse and commercial human DNA, TA cloned into pGL3-Promoter vector (Promega). The sequence cloned into pGL3 was confirmed by sequencing. Luciferase assays were carried out as previously described [65].

\section{Additional material}

Additional file $\mathbf{1}$ L-R micro array data. The top asymmetric expressing genes as indicated by the micro-array analysis. Data from 4 experiments has been averaged and genes ranked for stronger left (top) or right (bottom) sided expression. Genes subsequently analysed by in situ are highlighted.

Additional file 2 ASE containing sequences around Ablim 1. Sequences containing two or more FoxH1 binding sites (TGT G/T T/G ATT) within a 30$200 \mathrm{bp}$ region, in and surrounding the mouse and human Ablim 1 loci.

\section{Abbreviations}

ASE: asymmetric element; EST: expressed sequence tag; LPM: Lateral plate mesoderm; L-R: Left-Right; UTR: untranslated region; VHP: villin headpiece; WISH: wholemount in situ hybridisation. 


\section{Authors' contributions}

JS carried out the majority of dissections, genotyping and molecular genetic analysis. AE carried out additional practical work and contributed to experimental design. $\mathrm{HH}$ and PU performed and analysed the microarray experiment. JB and SB planned and performed the luciferase analysis. NB provided genotyped mutant embryos for analysis. DN conceived, designed and supervised the project and wrote the manuscript. All authors read and approved the final manuscript.

\section{Acknowledgements}

We would like to thank Aimee Ryan for discussion of the data and critical reading of the manuscript. Debbie Williams for submitting the microarray data to ArrayExpress. Jenny Murdoch, Andy Greenfield, Paraskevi Goggolidou and members of the laboratory for critical reading of the manuscript. NB is funded by BHF Programme Grant RG/03/012. DN is funded by the UK MRC. SB is funded by the Wellcome Trust and BHF.

\section{Author Details}

IMRC Mammalian Genetics Unit, MRC Harwell, Harwell Science and Innovation Campus, Oxfordshire, OX11 ORD, UK, ²Dept of Cardiovascular Medicine, University of Oxford, Wellcome Trust Centre for Human Genetics, Roosevelt Drive Headington Oxford OX3 7BN, UK, ${ }^{3}$ Division of Basic Medical Sciences, St George's University of London, Cranmer Terrace, London SW17 ORE, UK, ${ }^{4}$ Centre for Regenerative Medicine Chancellor's Building, 49 Little France Crescent, Edinburgh EH16 4SB, UK, 5José Bragança Departamento de Ciências Biomédicas e Medicina, Universidade do Algarve, Campus de Gambelas, 8005139 Faro, Portugal and ${ }^{6} \mid B B-I n s t i t u t e$ for Biotechnology and Bioengineering, Centro de Biomedicina Molecular e Estrutural, UAlg. Portugal

Received: 25 August 2009 Accepted: 20 May 2010

Published: 20 May 2010

\section{References}

1. Ramsdell AF: Left-right asymmetry and congenital cardiac defects: Getting to the heart of the matter in vertebrate left-right axis determination. Dev Biol 2005:1-20.

2. Aylsworth AS: Clinical aspects of defects in the determination of laterality. Am J Med Genet 2001, 101:345-55

3. Badano JL, Mitsuma N, Beales PL, Katsanis N: The Ciliopathies: An Emerging Class of Human Genetic Disorders. Annual Review of Genomics and Human Genetics 2006, 7:125-148.

4. Shiratori $H$, Hamada $H$ : The left-right axis in the mouse: from origin to morphology. Development 2006, 133:2095-104.

5. Nonaka S, Shiratori H, Saijoh Y, Hamada H: Determination of left-right patterning of the mouse embryo by artificial nodal flow. Nature 2002, 418:96-9.

6. Nonaka S, Tanaka Y, Okada Y, Takeda S, Harada A, Kanai Y, Kido M, Hirokawa N: Randomization of left-right asymmetry due to loss of nodal cilia generating leftward flow of extraembryonic fluid in mice lacking KIF3B motor protein. Cell 1998, 95:829-37.

7. McGrath J, Somlo S, Makova S, Tian X, Brueckner M: Two populations of node monocilia initiate left-right asymmetry in the mouse. Cell 2003, 114:61-73.

8. Tanaka Y, Okada Y, Hirokawa N: FGF-induced vesicular release of Sonic hedgehog and retinoic acid in leftward nodal flow is critical for leftright determination. Nature 2005, 435:172-177.

9. Norris D: Breaking the left-right axis: do nodal parcels pass a signal to the left? Bioessays 2005, 27:991-4.

10. Hamada H, Meno C, Watanabe D, Saijoh Y: Establishment of vertebrate left-right asymmetry. Nat Rev Genet 2002, 3:103-13.

11. Logan M, Pagan-Westphal S, Smith DM, Paganessi L, Tabin CJ: The transcription factor Pitx2 mediates situs-specific morphogenesis in response to left-right asymmetric signals. Cell 1998, 94:307-17.

12. Ryan RK, Blumberg B, Rodriguez-Esteban C, Yonei-Tamura S, Tamura K, Tsukui T, de la Pena J, Sabbagh W, Greenwald J, Choe S, et al:: Pitx2 determines left-right asymmetry of internal organs in vertebrates. Nature 1998, 394:545-51

13. Lu C, Huang X, Ma HF, Gooley JJ, Aparacio J, Roof DJ, Chen C, Chen DF, Li $T$ : Normal retinal development and retinofugal projections in mice lacking the retina-specific variant of actin-binding LIM domain protein. Neuroscience 2003, 120:121-31.
14. Collignon J, Varlet I, Robertson EJ: Relationship between asymmetric nodal expression and the direction of embryonic turning [see comments]. Nature 1996, 381:155-8.

15. Lowe LA, Supp DM, Sampath K, Yokoyama T, Wright CV, Potter SS, Overbeek P, Kuehn MR: Conserved left-right asymmetry of nodal expression and alterations in murine situs inversus. Nature 1996, 381:158-61

16. Hummel KP, Chapman DB: Visceral inversion and associated anomalies in the mouse. J Hered 1955:9-13.

17. Layton WM Jr: Random determination of a developmental process: reversal of normal visceral asymmetry in the mouse. J Hered 1976, 67:336-8.

18. Okada Y, Nonaka S, Tanaka Y, Saijoh Y, Hamada H, Hirokawa N: Abnormal nodal flow precedes situs inversus in iv and inv mice. Mol Cell 1999, 4:459-68.

19. Tsukui T, Capdevila J, Tamura K, Ruiz-Lozano P, Rodriguez-Esteban C, Yonei-Tamura S, Magallon J, Chandraratna RA, Chien K, Blumberg B, et al.: Multiple left-right asymmetry defects in Shh(-/-) mutant mice unveil a convergence of the shh and retinoic acid pathways in the control of Lefty-1. Proc Natl Acad Sci USA 1999, 96:11376-81.

20. Norris DP, Brennan J, Bikoff EK, Robertson EJ: The Foxh1-dependent autoregulatory enhancer controls the level of Nodal signals in the mouse embryo. Development 2002, 129:3455-68.

21. Norris D, Robertson EJ: Asymmetric and node-specific nodal expression patterns are controlled by two distinct cis-acting regulatory elements. Genes Dev 1999, 13:1575-88.

22. Liu C, Liu W, Palie J, Lu MF, Brown NA, Martin JF: Pitx2c patterns anterior myocardium and aortic arch vessels and is required for local cell movement into atrioventricular cushions. Development 2002, 129:5081-91.

23. Brennan J, Norris DP, Robertson EJ: Nodal activity in the node governs left-right asymmetry. Genes Dev 2002, 16:2339-44.

24. Shiratori H, Sakuma R, Watanabe M, Hashiguchi H, Mochida K, Sakai Y, Nishino J, Saijoh Y, Whitman M, Hamada H: Two-step regulation of leftright asymmetric expression of Pitx2: initiation by nodal signaling and maintenance by Nkx2. Mol Cell 2001, 7:137-49.

25. Campione M, Steinbeisser $H$, Schweickert A, Deissler K, van Bebber F, Lowe LA, Nowotschin S, Viebahn C, Haffter P, Kuehn MR, et al: The homeobox gene Pitx2: mediator of asymmetric left-right signaling in vertebrate heart and gut looping. Development 1999, 126:1225-34.

26. Gage PJ, Suh H, Camper SA: Dosage requirement of Pitx2 for development of multiple organs. Development 1999, 126:4643-51.

27. Kitamura K, Miura H, Miyagawa-Tomita S, Yanazawa M, Katoh-Fukui Y, Suzuki R, Ohuchi H, Suehiro A, Motegi Y, Nakahara Y, et al.: >Mouse Pitx2 deficiency leads to anomalies of the ventral body wall, heart, extraand periocular mesoderm and right pulmonary isomerism. Development 1999, 126:5749-58.

28. Lin CR, Kioussi C, O'Connell S, Briata P, Szeto D, Liu F, Izpisua-Belmonte JC, Rosenfeld MG: Pitx2 regulates lung asymmetry, cardiac positioning and pituitary and tooth morphogenesis. Nature 1999, 401:279-82.

29. Bamford RN, Roessler E, Burdine RD, Saplakoglu U, dela Cruz J, Splitt M, Goodship JA, Towbin J, Bowers P, Ferrero GB, et al: Loss-of-function mutations in the EGF-CFC gene CFC1 are associated with human leftright laterality defects. Nat Genet 2000, 26:365-9.

30. Mine M, Anderson RM, Klingensmith J: BMP antagonism is required in both the node and lateral plate mesoderm for mammalian left-right axis establishment. Development 2008, 135:2425-34

31. Schneider A, Mijalski T, Schlange T, Dai W, Overbeek P, Arnold HH, Brand T: The homeobox gene NKX3.2 is a target of left-right signalling and is expressed on opposite sides in chick and mouse embryos. Curr Biol 1999, 9:911-4.

32. Murray SA, Gridley T: Snail family genes are required for left-right asymmetry determination, but not neural crest formation, in mice. Proc Natl Acad Sci USA 2006, 103:10300-4

33. Nieto MA, Bennett MF, Sargent MG, Wilkinson DG: Cloning and developmental expression of Sna, a murine homologue of the Drosophila snail gene. Wilkinson 1992, 116:227-37.

34. Hou H, Yashiro K, Okazaki Y, Saijoh Y, Hayashizaki Y, Hamada H Identification of a novel left-right asymmetrically expressed gene in the mouse belonging to the BPI/PLUNC superfamily. Dev Dyn 2004 229:373-9. 
35. Marques S, Borges AC, Silva AC, Freitas S, Cordenonsi M, Belo JA: The activity of the Nodal antagonist Cerl-2 in the mouse node is required for correct L/R body axis. Genes Dev 2004, 18:2342-7.

36. Pearce JJ, Penny G, Rossant J: A mouse cerberus/Dan-related gene family. Dev Biol 1999, 209:98-110.

37. Gros J, Feistel K, Viebahn C, Blum M, Tabin CJ: Cell movements at Hensen's node establish left/right asymmetric gene expression in the chick. Science 2009, 324:941-4.

38. Cui C, Little CD, Rongish BJ: Rotation of organizer tissue contributes to left-right asymmetry. Anat Rec (Hoboken) 2009, 292:557-61.

39. Yamanaka Y, Tamplin OJ, Beckers A, Gossler A, Rossant J: Live imaging and genetic analysis of mouse notochord formation reveals regional morphogenetic mechanisms. Dev Cell 2007, 13:884-96.

40. Sulik K, Dehart DB, langaki T, Carson JL, Vrablic T, Gesteland K, Schoenwolf GC: Morphogenesis of the murine node and notochordal plate. Dev Dyn 1994, 201:260-78.

41. Lee JD, Anderson KV: Morphogenesis of the node and notochord: the cellular basis for the establishment and maintenance of left-right asymmetry in the mouse. Dev Dyn 2008, 237:3464-76

42. Cartwright JH, Piro N, Piro O, Tuval I: Fluid dynamics of nodal flow and left-right patterning in development. Dev Dyn 2008, 237:3477-90.

43. Essner JJ, Vogan KJ, Wagner MK, Tabin CJ, Yost HJ, Brueckner M: Conserved function for embryonic nodal cilia. Nature 2002, 418:37-8.

44. Schweickert A, Weber T, Beyer T, Vick P, Bogusch S, Feistel K, Blum F: Ciliadriven leftward flow determines laterality in Xenopus. Curr Bio/ 2007, 17:60-6.

45. Blum M, Weber T, Beyer T, Vick P: Evolution of leftward flow. Semin Cell Dev Biol 2008, 20:464-71.

46. Hyatt BA, Lohr JL, Yost HJ: Initiation of vertebrate left-right axis formation by maternal Vg1. Nature 1996, 384:62-5.

47. Hyatt BA, Yost HJ: The left-right coordinator: the role of $\mathrm{Vg} 1$ in organizing left-right axis formation. Cell 1998, 93:37-46.

48. Kramer KL, Barnette JE, Yost HJ: PKCgamma regulates syndecan-2 insideout signaling during xenopus left-right development. Cell 2002, 111:981-90

49. Kramer KL, Yost HJ: Ectodermal syndecan-2 mediates left-right axis formation in migrating mesoderm as a cell-nonautonomous $\mathrm{Vg} 1$ cofactor. Dev Cell 2002, 2:115-24.

50. Bunney TD, De Boer AH, Levin M: Fusicoccin signaling reveals 14-3-3 protein function as a novel step in left-right patterning during amphibian embryogenesis. Development 2003, 130:4847-58.

51. Adams DS, Robinson KR, Fukumoto T, Yuan S, Albertson RC, Yelick P, Kuo L, McSweeney M, Levin M: Early, H+-V-ATPase-dependent proton flux is necessary for consistent left-right patterning of non-mammalian vertebrates. Development 2006, 133:1657-71.

52. Levin M, Thorlin T, Robinson KR, Nogi T, Mercola M: Asymmetries in $\mathrm{H}+$ / $\mathrm{K}+$-ATPase and cell membrane potentials comprise a very early step in left-right patterning. Cell 2002, 111:77-89.

53. Esser AT, Smith KC, Weaver JC, Levin M: Mathematical model of morphogen electrophoresis through gap junctions. Dev Dyn 2006, 235:2144-59.

54. Fukumoto T, Kema IP, Levin M: Serotonin signaling is a very early step in patterning of the left-right axis in chick and frog embryos. Curr Biol 2005, 15:794-803

55. Oki S, Hashimoto R, Okui Y, Shen MM, Mekada E, Otani H, Saijoh Y, Hamada H: Sulfated glycosaminoglycans are necessary for Nodal signal transmission from the node to the left lateral plate in the mouse embryo. Development 2007, 134:3893-904.

56. Roof DJ, Hayes A, Adamian M, Chishti AH, Li T: Molecular characterization of abLIM, a novel actin-binding and double zinc finger protein. J Cell Biol 1997, 138:575-88.

57. Struckhoff EC, Lundquist EA: The actin-binding protein UNC-115 is an effector of Rac signaling during axon pathfinding in C. elegans. Development 2003, 130:693-704.

58. Lundquist EA, Herman RK, Shaw JE, Bargmann Cl: UNC-115, a conserved protein with predicted LIM and actin-binding domains, mediates axon guidance in C. elegans. Neuron 1998, 21:385-92.

59. Erkman L, Yates PA, McLaughlin T, McEvilly RJ, Whisenhunt T, O'Connell SM, Krones Al, Kirby MA, Rapaport DH, Bermingham JR, et al:: A POU domain transcription factor-dependent program regulates axon pathfinding in the vertebrate visual system. Neuron 2000, 28:779-92.
60. Yang $Y$, Lundquist EA: The actin-binding protein UNC-115/abLIM controls formation of lamellipodia and filopodia and neuronal morphogenesis in Caenorhabditis elegans. Mol Cell Biol 2005, 25:5158-70

61. Hummel KP, Chapman DB: Visceral inversion and associated anomalies in the mouse. JHered 1959:9-13.

62. Supp DM, Witte DP, Potter SS, Brueckner M: Mutation of an axonemal dynein affects left-right asymmetry in inversus viscerum mice. Nature 1997, 389:963-6.

63. Ermakov A, Stevens JL, Whitehill E, Robson JE, Pieles G, Brooker D, Goggolidou P, Powles-Glover N, Hacker T, Young SR, et al:: Mouse mutagenesis identifies novel roles for left-right patterning genes in pulmonary, craniofacial, ocular, and limb development. Dev Dyn 2009, 238:581-94

64. Chiang $C$, Litingtung $Y$, Lee $E$, Young KE, Corden JL, Westphal H, Beachy PA: Cyclopia and defective axial patterning in mice lacking Sonic hedgehog gene function. Nature 1996, 383:407-13.

65. Braganca J, Eloranta JJ, Bamforth SD, Ibbitt JC, Hurst HC, Bhattacharya S: Physical and functional interactions among AP-2 transcription factors, p300/CREB-binding protein, and CITED2. J Biol Chem 2003, 278:16021-9.

doi: 10.1186/1471-213X-10-54

Cite this article as: Stevens et al., Analysis of the asymmetrically expressed Ablim1 locus reveals existence of a lateral plate Nodal-independent left sided signal and an early, left-right independent role for nodal flow BMC Developmental Biology 2010, 10:54

\section{Submit your next manuscript to BioMed Centra and take full advantage of:}

- Convenient online submission

- Thorough peer review

- No space constraints or color figure charges

- Immediate publication on acceptance

- Inclusion in PubMed, CAS, Scopus and Google Scholar

- Research which is freely available for redistribution
C Biomed Central 